\title{
NNLO W Pair Production at the LHC: Status Report
}

\author{
Grigorios CHACHAMIS* ${ }^{* \dagger}$ \\ Instituto de Física Corpuscular, Universitat de València - Consejo Superior de Investigaciones \\ Científicas, Parc Científic, E-46980 Paterna (Valencia), Spain \\ E-mail: grigorios.chachamis@ific.uv.es
}

\begin{abstract}
We discuss the result with full mass dependence for the virtual NNLO QCD corrections to the $\mathrm{W}$ boson pair production in the quark-anti-quark annihilation channel. We also report on our progress regarding the treatment of the double-real radiative corrections which, along with the virtual-real corrections, are the other two necessary ingredients for a theoretical prediction of the total cross section for $\mathrm{W}^{+} \mathrm{W}^{-}$production to NNLO accuracy.
\end{abstract}

The European Physical Society Conference on High Energy Physics

18-24 July, 2013

Stockholm, Sweden

\footnotetext{
* Speaker.

${ }^{\dagger}$ The speaker wishes to thank the Institut für Theoretische Teilchenphysik und Kosmologie, RWTH Aachen, for hospitality during the latest stages of work on this project.
} 


\section{Introduction}

Diboson production plays a crucial role in the Large Hadron Collider (LHC) physics program. Any deviations of the measured cross sections from the Standard Model (SM) predictions would indicate new physics. The deviations could arise from anomalous triple vector boson interactions or from new particles decaying into gauge bosons. Furthermore, the measurements of WW and ZZ production cross sections are essential for Higgs boson studies.

In particular, $\mathrm{W}$ pair production in the quark-anti-quark annihilation channel,

$$
q \bar{q} \rightarrow \mathrm{W}^{+} \mathrm{W}^{-},
$$

plays an essential role as it serves as a signal process in the search for new physics and also is the dominant irreducible background to the Higgs poduction channel $p p \rightarrow \mathrm{H} \rightarrow \mathrm{W}^{*} \mathrm{~W}^{*} \rightarrow l \bar{v} \bar{l}^{\prime} v^{\prime}$. Both ATLAS [1] and CMS [2] collaborations have released measurements of the WW cross section at $\sqrt{s}=7 \mathrm{TeV}$ whereas CMS has also published data for $\sqrt{s}=8 \mathrm{TeV}$ [3]. The measured cross section for $\mathrm{W}$ boson pair production, by both collaborations, shows a tendency to be larger than the current theoretical prediction within the SM, a fact that calls in itself for more accurate theoretical predictions.

The process (1.1), initially calculated at leading order more that three decades ago [4], is currently known at next-to-leading order (NLO) accuracy in QCD [5, 6, 7, 8, 9, 10], after a tremendous effort. Soft-gluon resummation effects have been assessed in Refs. [11, 12] while the electroweak corrections are also known to NLO $[13,14,15,16]$. The NLO QCD corrections were proven to be large, enhancing the tree-level result by almost $70 \%$ which falls to a still large $30 \%$ after imposing a jet veto. Therefore, for a more accurate theoretical estimate -with un uncertainty of less than $10 \%$ - to be compared against the experimental measurements at the LHC, one is bound to go one order higher in the perturbative expansion, namely, to the next-to-next-to-leading order (NNLO).

To complete the picture, there is another process that needs to be considered and which is the $\mathrm{W}$ pair production in the loop-induced gluon fusion channel,

$$
g g \rightarrow \mathrm{W}^{+} \mathrm{W}^{-} .
$$

The latter contributes at $\mathscr{O}\left(\alpha_{s}^{2}\right)$ relative to the quark-anti-quark-annihilation channel but is nevertheless enhanced due to the large gluon flux at the LHC [17, 18].

Beyond NLO accuracy in QCD, there are only partial results in the literature [19, 20, 21, 22]. We have computed the NNLO two-loop [19] and the one-loop squared [20] virtual corrections in the high energy limit few years ago. However, this is not enough to cover the kinematical region close to threshold. Therefore, in order to cover all kinematical regions we proceed as follows. We perform a deep expansion in the $\mathrm{W}$ mass around the high energy limit which in combination with the method of numerical integration of differential equations [23, 24, 25] allows us the numerical computation of the two-loop ${ }^{1}$ amplitude with full mass dependence over the whole phase space. We report our progress on that as well as on the computation of the double-real NNLO corrections in the following Sections.

\footnotetext{
${ }^{1}$ We work in a similar fashion for the one-loop squared virtual corrections. However, here we restrict the discussion to the two-loop corrections only.
} 


\section{The virtual NNLO corrections}

The method for computing the amplitude in the high energy limit, namely the limit where $s$ is much larger than the $\mathrm{W}$ mass, $m$, is similar to the one followed in Refs. [26, 27]. The amplitude is reduced such that it only contains a small number of integrals (master integrals) by use of the Laporta algorithm [28]. Next comes the derivation, in a fully automatized way, of the MellinBarnes (MB) representations [29, 30] of all the master integrals by using the MATHEMATICA package MBREPRESENTATION [31]. The MB representations are in turn analytically continued in the number of space-time dimensions by means of the MB package [32], thus revealing the full singularity structure. An asymptotic expansion in the mass is performed by closing contours and the integrals are finally resummed, either with the help of XSUMMER [33] or the PSLQ algorithm [34]. The final result is expressed in terms of harmonic polylogarithms.

The high energy limit result by itself is not enough, as was mentioned before. The next step, following the methods applied in Ref. [35], is to compute power corrections in the W mass. Power corrections are good enough to cover most of the phase space, apart from the region near threshold as well as the regions corresponding to small angle scattering.

We introduce here some of the notation of Ref. [19] for completeness. The W pair production process in the quark-anti-quark annihilation channel reads in detail:

$$
q\left(p_{1}\right)+\bar{q}\left(p_{2}\right) \rightarrow \mathrm{W}^{-}\left(p_{3}, m\right)+\mathrm{W}^{+}\left(p_{4}, m\right),
$$

where $p_{i}$ denote the quark and $\mathrm{W}$ momenta.

We choose to express the amplitude in terms of the kinematic variables $x$ and $m_{s}$ which are defined to be

$$
x=-\frac{t}{s}, m_{s}=\frac{m^{2}}{s}
$$

where

$$
s=\left(p_{1}+p_{2}\right)^{2} \text { and } t=\left(p_{1}-p_{3}\right)^{2}-m^{2} .
$$

The variation then of $x$ within the range $[1 / 2(1-\beta), 1 / 2(1+\beta)]$, where $\beta=\sqrt{1-4 m^{2} / s}$ is the velocity, corresponds to angular variation between the forward and backward scattering.

Any master integral $M_{i}$ can be written then as

$$
M_{i}=M_{i}\left(m_{s}, x, \varepsilon\right)=\sum_{j=k}^{l} \varepsilon^{j} I_{i j}\left(m_{s}, x\right),
$$

where $\varepsilon$ is the usual regulator in dimensional regularization $(d=4-2 \varepsilon)$ and the lowest power of $\varepsilon$ in the sum can be -4 .

The key point now, is that the derivative of any Feynman integral with respect to any kinematical variable is again a Feynman integral with denominators or numerators raised to some new powers and which may be reduced anew in terms of the initial set of master integrals. This way, one can construct a partially triangular system of differential equations in $m_{s}$, which can subsequently be solved in the form of a power series expansion in $m_{s}$.

If we differentiate an arbitrary master integral, $M_{i}\left(m_{s}, x, \varepsilon\right)$, with respect to $m_{s}$ and $x$, we will have respectively

$$
m_{s} \frac{d}{d m_{s}} M_{i}\left(m_{s}, x, \varepsilon\right)=\sum_{j} C_{i j}\left(m_{s}, x, \varepsilon\right) M_{j}\left(m_{s}, x, \varepsilon\right)
$$


and

$$
x \frac{d}{d x} M_{i}\left(m_{s}, x, \varepsilon\right)=\sum_{j} C_{i j}^{\prime}\left(m_{s}, x, \varepsilon\right) M_{j}\left(m_{s}, x, \varepsilon\right) .
$$

We use Eq. (2.5) to obtain the mass corrections for the master integrals calculating the power series expansion up to order $m_{s}^{30}$ (see also Ref. [35] for more details). This deep expansion in $m_{s}$ should be sufficient for most of the phase space but still not enough to cover the whole allowed kinematical region. From this point on, the way to proceed is by numerically integrating the system of differential equations. We choose to work with the master integrals in the form of Eq. (2.4), where the $\varepsilon$-dependence is explicit. We can then work with the coefficients of the $\varepsilon$ terms and accordingly have

$$
m_{s} \frac{d}{d m_{s}} I_{i}\left(m_{s}, x\right)=\sum_{j} J_{i j}^{M}\left(m_{s}, x\right) I_{j}\left(m_{s}, x\right)
$$

and

$$
x \frac{d}{d x} I_{i}\left(m_{s}, x\right)=\sum_{j} J_{i j}^{X}\left(m_{s}, x\right) I_{j}^{\prime}\left(m_{s}, x\right)
$$

where the Jacobian matrices $J^{M}$ and $J^{X}$ have as elements rational functions.

By using the system of differential equations $(2.7,2.8)$, one can obtain a numerical solution for the amplitude. Actually, what we are dealing now with is an initial value problem and the main requirement is to have the initial conditions to high enough accuracy. The initial conditions are nothing but the values of the master integrals at a proper kinematical point, which we call initial point, and can be provided by the power series expansion. The initial point has to be chosen somewhere in the high energy limit region, where $m_{s}$ is small and therefore, the values obtained by the power series are very accurate. Starting from there, one can evolve to any other point of the phase space after integrating numerically the system of differential equations $(2.7,2.8)$.

We parametrize with a suitable grid of points the region close to threshold and then we calculate the master integrals for the points of the grid by evolving as described above. Given that the master integrals have to be very smooth, one can use interpolation, after having the values for the grid points, in order to get the values at any point of the region. We use 1600 points for the grid and take as initial conditions the values of the master integrals at the point $m_{s}=5 \times 10^{-3}, x=1 / 4$.

The numerical integration is performed by using ODEPACK [36], one of the most advanced software packages implementing the variable coefficient multistep method. We use quadruple precision to maximize accuracy. The values at any single grid point can be obtained in about 10 minutes in average (with a typical $2.5 \mathrm{GHz}$ Intel Core 2 Duo system) after compilation with the INTEL FORTRAN COMPILER. The achieved accuracy is beyond 10 digits for most of the points of the grid. It is also worth noting that in order to perform the numerical integration one needs to deform the contour in the complex plane away from the real axis. This is due to the fact that along the real axis there are spurious singularities. We use an elliptic contour and we achieve a better estimate of the final global error by calculating more than once for each point of the grid, using each time different eccentricities. We will not present here any numbers since the aim was to report on the general methods. The details and the results of the study will be presented in a future publication [37]. 


\section{The double-real NNLO corrections}

In this section we will only describe our methodology as this is ongoing work, the results will be given in a following publication.

To begin with, the matrix elements for the double-real NNLO QCD corrections are needed and these can be obtained by the following processes:

$$
\begin{gathered}
0 \rightarrow u\left(p_{1}\right)+\bar{u}\left(p_{2}\right)+\mathrm{W}^{-}\left(p_{3}, m\right)+\mathrm{W}^{+}\left(p_{4}, m\right)+g\left(p_{5}\right)+g\left(p_{6}\right), \\
0 \rightarrow u\left(p_{1}\right)+\bar{u}\left(p_{2}\right)+\mathrm{W}^{-}\left(p_{3}, m\right)+\mathrm{W}^{+}\left(p_{4}, m\right)+q^{\prime}\left(p_{5}\right)+\bar{q}^{\prime}\left(p_{6}\right), \\
0 \rightarrow d\left(p_{1}\right)+\bar{d}\left(p_{2}\right)+\mathrm{W}^{-}\left(p_{3}, m\right)+\mathrm{W}^{+}\left(p_{4}, m\right)+g\left(p_{5}\right)+g\left(p_{6}\right), \\
0 \rightarrow d\left(p_{1}\right)+\bar{d}\left(p_{2}\right)+\mathrm{W}^{-}\left(p_{3}, m\right)+\mathrm{W}^{+}\left(p_{4}, m\right)+q^{\prime}\left(p_{5}\right)+\bar{q}^{\prime}\left(p_{6}\right),
\end{gathered}
$$

where $u$ denotes an up-type quark and $d$ a down-type one, if we assume all possible crossings of quarks and gluons in the initial state, such that we end up with a $2 \rightarrow 4$ process while the $\mathrm{W}$ pair remains in the final state. Similarly, the NLO real matrix elements are given by crossings to the processes:

$$
\begin{aligned}
& 0 \rightarrow u\left(p_{1}\right)+\bar{u}\left(p_{2}\right)+\mathrm{W}^{-}\left(p_{3}, m\right)+\mathrm{W}^{+}\left(p_{4}, m\right)+g\left(p_{5}\right), \\
& 0 \rightarrow d\left(p_{1}\right)+\bar{d}\left(p_{2}\right)+\mathrm{W}^{-}\left(p_{3}, m\right)+\mathrm{W}^{+}\left(p_{4}, m\right)+g\left(p_{5}\right),
\end{aligned}
$$

whereas the LO one is given by Eq. (2.1) for q being either an up-type quark or a down-type one. We generate the matrix elements using FEYNARTS [38], we simplify them with FORM [39] and, as a cross-check, we compare against MADGRAPH [40].

With the matrix elements at hand, we use the general subtraction scheme STRIPPER [41] for the evaluation of the NNLO QCD contributions from double-real radiation. The result is a Laurent expansion in the parameter of dimensional regularization, the coefficients of which are evaluated by numerical Monte Carlo integration. The two main ideas behind STRIPPER are a two-level decomposition of the phase space, the second one factorizing the singular limits of amplitudes, and a suitable parameterization of the kinematics allowing for derivation of subtraction and integrated subtraction terms from eikonal factors and splitting functions without non-trivial analytic integration.

\section{Conclusions}

The production of a $\mathrm{W}^{+} \mathrm{W}^{-}$pair via quark-anti-quark-annihilation is an important process in the LHC physics program for which higher accuracy theoretical estimates are essential. After having calculated the two-loop and the one-loop-squared virtual QCD corrections to the $\mathrm{W}$ boson pair production in the high energy limit, we described how we use a combination of a deep expansion in the $\mathrm{W}$ mass around the high energy limit and numerical integration of differential equations to compute the virtual amplitudes with full mass dependence over the whole phase space. Finally, we presented our methodology for evaluating the double-real NNLO QCD corrections. 


\section{Acknowledgements}

This work has been supported by Marie Curie Actions (PIEF-GA-2011-298582) and in part by the Spanish Government and ERDF funds from the EU Commission [Grants No. FPA2011-23778, No. CSD2007-00042 (Consolider Project CPAN)] and by Generalitat Valenciana under Grant No. PROMETEOII/2013/007.

\section{References}

[1] G. Aad et al. [ATLAS Collaboration], Phys. Rev. D 87, 112001 (2013) [arXiv:1210.2979 [hep-ex]].

[2] S. Chatrchyan et al. [CMS Collaboration], Phys. Lett. B 721, 190 (2013) [arXiv:1301.4698 [hep-ex]].

[3] S. Chatrchyan et al. [CMS Collaboration], arXiv:1306.1126 [hep-ex].

[4] R. W. Brown and K. O. Mikaelian, Phys. Rev. D 19, 922 (1979).

[5] J. Ohnemus and J. F. Owens, Phys. Rev. D 43 (1991) 3626;

J. Ohnemus, Phys. Rev. D 44 (1991) 3477 and Phys. Rev. D 44 (1991) 1403;

B. Mele, P. Nason and G. Ridolfi, Nucl. Phys. B 357 (1991) 409;

S. Frixione, P. Nason and G. Ridolfi, Nucl. Phys. B 383 (1992) 3;

S. Frixione, Nucl. Phys. B 410 (1993) 280.

[6] U. Baur, T. Han and J. Ohnemus, Phys. Rev. D 53 (1996) 1098 [hep-ph/9507336];

L. J. Dixon, Z. Kunszt and A. Signer, Nucl. Phys. B 531 (1998) 3 [hep-ph/9803250];

L. J. Dixon, Z. Kunszt and A. Signer, Phys. Rev. D 60, 114037 (1999) [hep-ph/9907305].

[7] J. M. Campbell and R. K. Ellis, Phys. Rev. D 60 (1999) 113006 [hep-ph/9905386].

[8] S. Frixione and B. R. Webber, JHEP 0206 (2002) 029 [hep-ph/0204244];

P. Nason and G. Ridolfi, JHEP 0608 (2006) 077 [hep-ph/0606275].

[9] K. Hamilton, JHEP 1101 (2011) 009 [arXiv:1009.5391 [hep-ph]];

S. Höche, F. Krauss, M. Schönherr and F. Siegert, JHEP 1104 (2011) 024 [arXiv:1008.5399 [hep-ph]];

T. Melia, P. Nason, R. Rontsch and G. Zanderighi, JHEP 1111 (2011) 078 [arXiv:1107.5051

[hep-ph]];

R. Frederix et al., JHEP 1202 (2012) 099 [arXiv:1110.4738 [hep-ph]].

[10] M. Billoni, S. Dittmaier, B. Jager and C. Speckner, arXiv:1310.1564 [hep-ph].

[11] M. Grazzini, JHEP 0601 (2006) 095 [hep-ph/0510337].

[12] S. Dawson, I. M. Lewis and M. Zeng, arXiv:1307.3249 [hep-ph];

Y. Wang, C. S. Li, Z. L. Liu, D. Y. Shao and H. T. Li, arXiv:1307.7520 [hep-ph].

[13] E. Accomando, A. Denner and A. Kaiser, Nucl. Phys. B 706 (2005) 325 [hep-ph/0409247];

E. Accomando and A. Kaiser, Phys. Rev. D 73 (2006) 093006 [hep-ph/0511088].

[14] J. H. Kühn, F. Metzler, A. A. Penin and S. Uccirati, JHEP 1106 (2011) 143 [arXiv:1101.2563 [hep-ph]].

[15] A. Bierweiler, T. Kasprzik, H. Kühn and S. Uccirati, JHEP 1211 (2012) 093 [arXiv:1208.3147 [hep-ph]];

A. Bierweiler, T. Kasprzik and J. H. Kühn, arXiv:1305.5402 [hep-ph].

[16] J. Baglio, L. D. Ninh and M. M. Weber, arXiv:1307.4331 [hep-ph]. 
[17] D. A. Dicus, C. Kao and W. W. Repko, Phys. Rev. D 36 (1987) 1570;

E. W. N. Glover and J. J. van der Bij, Nucl. Phys. B 321 (1989) 561.

[18] T. Binoth, M. Ciccolini, N. Kauer and M. Krämer, JHEP 0503 (2005) 065 [hep-ph/0503094]; T. Binoth, M. Ciccolini, N. Kauer and M. Krämer, JHEP 0612 (2006) 046 [hep-ph/0611170];

T. Binoth, N. Kauer and P. Mertsch, arXiv:0807.0024 [hep-ph].

[19] G. Chachamis, Acta Phys. Polon. B 38 (2007) 3563 [arXiv:0710.3035 [hep-ph]];

G. Chachamis, M. Czakon and D. Eiras, JHEP 0812 (2008) 003 [arXiv:0802.4028 [hep-ph]].

[20] G. Chachamis, M. Czakon and D. Eiras, arXiv:0806.3043 [hep-ph].

[21] T. Gehrmann, L. Tancredi and E. Weihs, JHEP 1308, 070 (2013) [arXiv:1306.6344 [hep-ph]].

[22] F. Campanario, M. Rauch and S. Sapeta, arXiv:1309.7293 [hep-ph].

[23] M. Caffo, H. Czyz, S. Laporta and E. Remiddi, Nuovo Cim. A 111, 365 (1998) [arXiv:hep-th/9805118].

[24] R. Boughezal, M. Czakon and T. Schutzmeier, JHEP 0709, 072 (2007) [arXiv:0707.3090 [hep-ph]].

[25] M. Czakon and T. Schutzmeier, JHEP 0807, 001 (2008) [arXiv:0712.2762 [hep-ph]].

[26] M. Czakon, A. Mitov and S. Moch, Phys. Lett. B 651, 147 (2007) [arXiv:0705.1975 [hep-ph]].

[27] M. Czakon, A. Mitov and S. Moch, Nucl. Phys. B 798, 210 (2008) [arXiv:0707.4139 [hep-ph]].

[28] S. Laporta, Int. J. Mod. Phys. A 15, 5087 (2000) [arXiv:hep-ph/0102033].

[29] V. A. Smirnov, Phys. Lett. B 460, 397 (1999) [arXiv:hep-ph/9905323].

[30] J. B. Tausk, Phys. Lett. B 469, 225 (1999) [arXiv:hep-ph/9909506].

[31] G. Chachamis, M. Czakon, Publication in preparation.

[32] M. Czakon, Comput. Phys. Commun. 175, 559 (2006) [arXiv:hep-ph/0511200].

[33] S. Moch and P. Uwer, Comput. Phys. Commun. 174, 759 (2006) [arXiv:math-ph/0508008].

[34] H. R. P. Ferguson and D. H. Bailey Math. of Comput. 53, 649, (1989)

[35] M. Czakon, Phys. Lett. B 664, 307 (2008) [arXiv:0803.1400 [hep-ph]].

[36] P. N. Brown, G. D. Byrne and A. C. Hindmarsh SIAM J. Sci. Stat. Comput. 1038 (1989).

[37] G. Chachamis, M. Czakon, P. Fiedler, A. Mitov, Publication in preparation.

[38] T. Hahn, Comput. Phys. Commun. 140, 418 (2001) [hep-ph/0012260].

[39] J. A. M. Vermaseren, math-ph/0010025.

[40] J. Alwall, M. Herquet, F. Maltoni, O. Mattelaer and T. Stelzer, JHEP 1106, 128 (2011) [arXiv:1106.0522 [hep-ph]].

[41] M. Czakon, Phys. Lett. B 693, 259 (2010) [arXiv:1005.0274 [hep-ph]]; M. Czakon, Nucl. Phys. B 849, 250 (2011) [arXiv:1101.0642 [hep-ph]]. 